\title{
Zum Bekanntheitsgrad erwerbsrelevanter Phraseme des Deut- schen. Eine Untersuchung bei Germanistik-Studierenden an der Nationalen Kapodistrias-Universität Athen
}

\author{
Marios Chrissou und Evangelos Makos (Athen)
}

\begin{abstract}
It is a common place in the phraseodidactic research that collocational fluency is a significant factor of language fluency. Nevertheless, formulaic language is not focused systematically in the Common European Framework of Reference for Languages and in learning materials for German as a foreign language. The lack of transparency and fragmentary treatment of set phrases have a negative impact over the development of collocational fluency.

The present paper focuses on the extent that students of German Language and Literature at the National Kapodistrian University of Athens master high-frequency and common set phrases of German based on a questionnaire survey conducted. The results of the survey are discussed in terms of the deficiencies of institutional guidelines and learning materials. In line with this, proposals are formulated for the improvement of the institutional framework and the teaching of set phrases.
\end{abstract}

\section{$1 \quad$ Einführung}

Phraseme wurden in der Linguistik lange als sprachliche Eigenheiten mit Ausnahmecharakter behandelt, da sie paradigmatisch und syntagmatisch Restriktionen unterworfen sind, die freie Wortverbindungen nicht kennen. Neuere korpuslinguistische Forschungsarbeiten (Sinclair 2004, 2008 und Steyer 2013) zeigen, dass Formelhaftigkeit als linguistisches Merkmal den Sprachgebrauch auf verschiedenen stilistischen Ebenen und in verschiedenen Bereichen des sozialen Lebens konstituiert und somit zum natürlichen Sprachgebrauch gehört (Chrissou 2012: 53). Weist man formelhafter Sprache einen festen Platz im geschriebenen und gesprochenen Diskurs zu, so ist es aus sprachdidaktischer Sicht konsequent, den Anspruch auf eine gezielte und systematische Förderung der phraseologischen Kompetenz auf allen Lernstufen zu erheben. Nach dem Gemeinsamen Europäischen Referenzrahmen für Sprachen (Europarat 2001) bildet die rezeptive und produktive Beherrschung zielsprachlicher Phraseme ${ }^{1}$ einen festen Bestandteil der fremdsprachlichen Kompetenz. Zur Entwicklung dieser Kompetenzen im Rahmen des gesteuerten Fremdsprachenunterrichts bedarf es zunächst der fundierten Se-

\footnotetext{
${ }^{1}$ Im vorliegenden Beitrag wird in Anlehnung an Burger et al. (2007: 3) der Terminus „Phrasem“ für phraseologische Phänomene einheitlich verwendet.
}

Linguistik online 89, 2/18 - http://dx.doi.org/10.13092/lo.89.4272

CC by 3.0 
lektion eines phraseologischen Lexikonausschnitts mit hohem kommunikativem Wert im Sinne eines Grundwortschatzes, seiner nachvollziehbaren Anordnung in einer sinnvollen Progression, d. h. in einer geeigneten chronologischen Abfolge, zur Förderung der phraseologischen Kompetenz auf den einzelnen Sprachniveaus und seiner angemessenen didaktischen Aufbereitung in Lernmaterialien. Diesen Forderungen werden die institutionellen Richtlinien und die Lernmaterialien nur bedingt gerecht. Zum einen erfolgt die differenzierte Beschreibung der sukzessiven Entwicklung der phraseologischen Kompetenz von Sprachniveau zu Sprachniveau im Gemeinsamen Europäischen Referenzrahmen für Sprachen und in Profile Deutsch kaum transparent und distinktiv (Chrissou, i. E.). Zum anderen ist die Lage der aktuellen Lernmaterialien für Deutsch als Fremdsprache bezüglich der Selektion, Anordnung und didaktischen Aufbereitung von Phrasemen in der Regel mangelhaft.

Vor diesem Hintergrund wird im vorliegenden Beitrag die phraseologische Kompetenz von Studierenden der Auslandsgermanistik auf der Grundlage einer fragebogengeleiteten Aktion erfasst und zur defizitären Lage der institutionellen Richtlinien und Lernmaterialien in Beziehung gesetzt. Hierfür wird die Kenntnis kommunikativ wichtiger phraseologischer Lexik bei Germanistik-Studierenden im Fachbereich für deutsche Sprache und Literatur der Nationalen Kapodistrias-Universität Athen erhoben. Aus der Untersuchung ergeben sich Teilfragen, die sich auf die Selektion phraseologischer Einheiten in Lernmaterialien für Deutsch als Fremdsprache, auf den Zusammenhang von Phrasemkenntnis und Sprachniveau bzw. Umgebung der Sozialisation sowie auf den Einfluss der Muttersprache als signifikanter Größe auf die Phrasemkenntnis beziehen. Aus der Auswertung der Erhebungsdaten werden institutionell und didaktisch relevante Schlussfolgerungen abgeleitet.

\section{Das Germanistik-Studium an der Nationalen Kapodistrias-Universität Athen}

Fremdsprachen besitzen in Griechenland einen hohen Stellenwert. Das Beherrschen von Fremdsprachen wird positiv eingeschätzt und stellt eine wichtige Voraussetzung für die berufliche Qualifikation dar. Dabei haben die Schüler der fünften Schulklasse des staatlichen Bildungswesens die Möglichkeit, eine zweite Fremdsprache zu erlernen - Deutsch oder Französisch. Erste Fremdsprache ist nach wie vor Englisch, das in der dritten Schulklasse eingeführt wird. Eng an den Stellenwert des Deutschen als Fremdsprache ist in Griechenland das Germanistikstudium gebunden, das in den Fachbereichen für deutsche Sprache und Literatur der Philosophischen Fakultäten der Nationalen Kapodistrias-Universität Athen und der Aristoteles-Universität Thessaloniki angeboten wird. In beiden Fällen handelt es sich um einen Diplomstudiengang, der acht Studiensemester umfasst und sich in ein Grund- (1.-4. Semester) und ein Hauptstudium (5.-8. Semester) gliedert. Für das Studium in Athen werden jährlich etwa 150 Studierende neu immatrikuliert. Der Ausbau der Sprachkompetenz stellt eine wichtige Voraussetzung für den erfolgreichen Verlauf und Abschluss des Germanistik-Studiums dar, in dem bereits ab dem ersten Studiensemester die Lehrveranstaltungen in deutscher Sprache abgehalten werden. Aus diesem Grund ist das Kursprogramm zur Sprachpraxis in den ersten vier Studiensemestern von grundlegender Bedeutung, und sein erfolgreicher Abschluss Voraussetzung für den Übergang zum Hauptstudium. Ziel ist, durch ein integriertes Sprachfertigkeitstraining das Niveau C1 spätestens bis zum Abschluss des zweiten Studienjahres zu erreichen. Nach Studienabschluss geht die Mehrheit der Studienabsolventen in die Lehre von Deutsch als Fremdsprache im öffentlichen oder privaten Sektor.

ISSN 1615-3014 


\section{Zur Selektion eines phraseologischen Grundwortschatzes für den DaF-Unterricht}

Eine wichtige Voraussetzung für die angemessene Förderung der phraseologischen Kompetenz in der Unterrichtspraxis ist die fundierte Bestimmung eines phraseologischen Grundwortschatzes (Optimum bzw. Minimum) mit hohem kommunikativem Wert, der den Kommunikationsbedürfnissen der Lernenden gerecht wird. Nach dem Gemeinsamen Europäischen Referenzrahmen für Sprachen (Europarat 2001: 113, 148) ist die fundierte Auswahl phraseologischer Lexik Voraussetzung für die effiziente Gestaltung der Wortschatzarbeit. Sie sollte auf der Grundlage „sprachstatistischer Prinzipien“ erfolgen, die über die Vorkommensfrequenz phraseologischer Einheiten im Diskurs Aufschluss geben. Korpusdaten stellen seit dem Aufschwung der Korpuslinguistik eine reliable Alternative zur Intuition von Linguisten und Didaktikern dar, so dass die Behandlung von Phrasemen im Unterricht nicht „dem Zufall überlassen“ wird oder ,,abhängig vom persönlichen Interesse des Lehrers“ (Hessky 2007: 12) erfolgt. „Ein phraseologisches Minimum oder auch Optimum könnte“ Ettinger (2007: 902) zufolge ,verhindern, dass nur sporadisch in der Sprachwirklichkeit auftretende Phraseme, die oftmals selbst von Muttersprachlern kaum verwendet werden, ausgiebig in Phrasemsammlungen eingeübt werden, und ein solches Minimum könnte andererseits auch eine sinnvolle Lernprogression ermöglichen“. Neben dem quantitativen Auswahlkriterium der Vorkommenshäufigkeit wird in der Phraseodidaktik das qualitative Kriterium der Geläufigkeit hervorgehoben (Hessky 2007: 14; Bubenhofer/Ptashnyk 2010: 14). Auf dieser Grundlage unterbreiten Hallsteinsdóttir et al. (2006) einen Vorschlag für einen phraseologischen Grundwortschatz. Dafür extrahieren sie vorwiegend Idiome und Teilidiome aus gängigen Lernerwörterbüchern für Deutsch als Fremdsprache, Übungsbüchern und Lexika zur Phraseologie des Deutschen und untersuchen die Vorkommensfrequenz von 1'112 Phrasemen im Korpus „Deutscher Wortschatz“ der Universität Leipzig. Die gewonnenen Korpusdaten überprüfen sie auf der Grundlage des intersubjektiven Kriteriums der Geläufigkeit, indem sie Muttersprachler des Deutschen zur Geläufigkeit der erfassten Phraseme befragen. Das Ergebnis ist eine nuancierte Liste, deren Kernbereich aus insgesamt 624 Phrasemen besteht, wobei 142 Einheiten davon eine besonders hohe Vorkommensfrequenz und Geläufigkeit aufweisen. Auch wenn es sich dabei um keinen abgeschlossenen, sondern um einen offenen, erweiterbaren Grundwortschatz handelt (Hallsteinsdóttir et al. 2006: 129), der durch weitere Untersuchungen zu erweitern bzw. zu differenzieren ist, ist davon auszugehen, dass er eine Teilmenge der häufigsten und geläufigsten Phraseme des Deutschen erfasst. Insofern stellt er einen phraseologischen Ausschnitt mit hoher Erwerbsrelevanz dar, der für den Einsatz im Unterricht Deutsch als Fremdsprache geeignet ist.

\section{$4 \quad$ Institutionelle Richtlinien und Lernmaterialien}

Es ist Aufgabe des Gemeinsamen Europäischen Referenzrahmens für Sprachen und seines Referenzwerks Profile Deutsch festzulegen, welche sprachlichen Anforderungen an die Lernenden gestellt werden und insbesondere welche Lexik auf welchem Sprachniveau und in welchem Beherrschungsmodus zum Unterrichtsgegenstand werden sollte. Der Erwerb von 
Phrasemen ${ }^{2}$ wird im Gemeinsamen Europäischen Referenzrahmen für Sprachen in Zusammenhang mit der lexikalischen, soziolinguistischen und pragmatischen Kompetenz sowie in Zusammenhang mit den einzelnen kommunikativen Aktivitäten thematisiert. Nach den entsprechenden Kannbeschreibungen beherrschen die Lernenden auf dem A-Niveau relativ wenige Idiome, um Informationen zur Person oder zu Personen aus dem unmittelbaren Umfeld, $\mathrm{zu}$ Orten und Zeitpunkten oder zu einfachen Alltagssituationen auszutauschen (Europarat 2001). Die Lernenden auf dem B-Niveau zeigen ausreichende Kenntnisse im Umgang mit frequenten Wendungen, die im Sprachgebrauch in Zusammenhang mit eher vorhersehbaren Situationen vorkommen (ibd.). Auch wenn sich die idiomatische Kompetenz nach dem Gemeinsamen Europäischen Referenzrahmen für Sprachen und nach Profile Deutsch vorwiegend ab dem B2-Niveau allmählich entwickelt (zuerst rezeptiv, später auch produktiv), kommen kommunikativ wichtige Phraseme durchaus auf dem A-Niveau, z. B. wie viel Uhr ist es?, wie spät ist es?, wie geht es dir?, spazieren gehen, Spaß machen. Das Beherrschen der pragmatischen Gebrauchsbedingungen eines großen Spektrums frequenter Idiome (richtige Einschätzung von Register bzw. Registerwechsel und Konnotationen) ist in rezeptiver bzw. produktiver Hinsicht an das C-Niveau gebunden (ibd.).

Auch wenn der Gemeinsame Europäische Referenzrahmen für Sprachen und Profile Deutsch eine Orientierungshilfe bieten, bleibt ihr Beitrag unsystematisch und kaum differenziert, „da sie den Lernfortschritt auf den einzelnen Niveaus nur rudimentär und ohne die erwartete Trennschärfe reflektieren“ (Chrissou, i. E.). Zudem sind sie fragmentarisch, da sie sich vorwiegend auf die Niveaus A1-B1 verteilen, während sich relativ wenige auf die Niveaus B2C2 beziehen (ibd.). Die wenig transparente und distinktive Beschreibung des sukzessiven Lernzuwachses im Bereich der Phraseologie auf den einzelnen Sprachniveaus spiegelt sich in den gängigen Lernmaterialien für Deutsch als Fremdsprache wider. In Anlehnung an die einschlägige Literatur ${ }^{3}$ ist zu konstatieren, dass es an geeigneten Lernmaterialien, die sich an den Ergebnissen der phraseodidaktischen Forschung orientieren, mangelt. Die Auswahl der Phraseme erfolgt eher zufällig und ihre didaktische Aufbereitung häufig nicht textorientiert. Zudem wird der handlungsorientierte Einsatz von Strategien, die die Lernenden zur selbstständigen Erschließung von Phrasemen in Texten und zur pragmatisch angemessenen Kontextualisierung im Sprachgebrauch befähigen, didaktisch kaum fokussiert.

\section{$5 \quad$ Die Datenerhebung}

\subsection{Zur Methode}

Zur Feststellung der phraseologischen Kompetenz von Germanistik-Studierenden fand während des Wintersemesters 2015/16 im Rahmen des Seminars „Lexikologie und Wortschatzdidaktik“ des Postgraduiertenstudiengangs „Schnittstellen zwischen Linguistik und Sprachdidaktik“ des Fachbereichs für deutsche Sprache und Literatur der Nationalen Kapodistrias-

\footnotetext{
2 Die im Gemeinsamen Europäischen Referenzrahmen für Sprachen unsystematisch verwendeten Termini wie „Satzformeln“, „,idiomatische Wendungen“, „,feststehende Muster“ bzw. „Phrasen“, „feste Kollokationen“, „Redewendungen“, „Aussprüche“, „Zitate“ und „,sprichwörtliche Redensarten“ für phraseologische Phänomene (Europarat 2001: 111, 112,120) suggerieren eine unnötige Komplexität, die der aktuellen Phraseologieforschung nicht gerecht wird (Chrissou 2012: 22).

${ }^{3}$ Siehe hierzu Ettinger (2007: 901) und Ďurčo (2007: 169).
} 
Universität Athen eine Datenerhebung statt, an der drei Studentengruppen an zwei Tagen teilnahmen. ${ }^{4}$ Insbesondere wurde eine schriftliche Befragung von 67 Studierenden, die sich vorwiegend im Grundstudium befanden, im Rahmen einer fragebogengeleiteten Aktion durchgeführt. Die Zusammenstellung der Stichprobe entstand nach dem Quotenverfahren, wobei die für die Untersuchung relevanten Merkmale der Gesamtheit der Studierenden entsprechen.

Die Vorteile der schriftlichen Befragung bestanden in der relativ schnell zu erreichenden Größe der Stichprobe und in der Beseitigung interferierender Faktoren (Interviewer-Effekte), die auf gegenseitigen Erwartungen basieren und das Antwortverhalten der Befragten in persönlichen Befragungen beeinflussen (Albert/Marx 2014: 62, 63). Da die durchgeführte Erhebung im Seminarraum und im Hörsaal standfand, hat sich die Fragebogenaktion hinsichtlich der Rücklaufquote, d. h. des Anteils der ausgefüllten und zurückgegebenen Fragebögen, bewährt (ibd.: 64).

Zur Beseitigung von Hemmungen beim Ausfüllen des Fragebogens wurden die befragten Studierenden in ihrer Muttersprache, d.h. Griechisch, ${ }^{5}$ schriftlich und mündlich über den Kontext und die Zielsetzung der fragebogengeleiteten Erhebung in Kenntnis gesetzt und über die Wahrung ihrer Anonymität versichert. Ziel war, ein lockeres Klima zu schaffen und somit keinen Leistungsdruck entstehen zu lassen, wie es in einer Prüfungssituation der Fall ist. Der Teil des Fragebogens, der sich auf den eigentlichen Untersuchungsgegenstand bezog, wurde in deutscher Sprache verfasst. Als Anlaufphase der Erhebung diente zunächst das Ausfüllen relevanter Sozialdaten zum Alter, Studiensemester, zertifizierten Sprachniveau, zur Umgebung und Sprache, in der die Studierenden sozialisiert wurden, ${ }^{6}$ sowie zu den Lehrwerken, mit denen sie Deutsch gelernt haben. Als Grundlage für die Gestaltung des eigentlichen Teils des Fragebogens diente der Kernbereich des phraseologischen Grundwortschatzes bzw. „Optimums“ nach Hallsteinsdóttir et al. (2006), dem die 20 häufigsten und geläufigsten Phraseme entnommen wurden und in den Fragebogen Eingang fanden. Diese Anzahl an Phrasemen erlaubte, die Länge des Fragebogens und der zu bearbeitenden Daten übersichtlich zu halten, und ermöglichte somit eine Fokussierung auf die zu ergänzenden Inhalte. Wiesen Phraseme lexikalische Varianten auf, wurden sie in der Form übernommen, die im phraseologischen Optimum von Hallsteinsdóttir et al. (2006) als erste genannt wird, wobei fakultative Elemente im phraseologischen Formativ weggelassen wurden. Zur Sicherstellung des selbstständigen Bearbeitens der Fragebögen und somit einer hohen Reliabilität der Erhebung kamen zwei Versionen desselben Fragebogens zum Einsatz: In der ersten Version wurden die Phraseme in absteigender Reihenfolge angeordnet, wobei das Phrasem mit der höchsten Vorkommenshäufigkeit und Geläufigkeit als erstes angeführt wurde, und in der zweiten Version wurden sie in umgekehrter Reihenfolge aufgelistet.

Der erste Teil des Fragebogens (Aufgabe A) hatte die Form einer Multiple-Choice-Aufgabe und verlangte von den Befragten die Ergänzung der korrekten Form der ausgewählten Phraseme, wobei jeweils der erste Teil des Phrasems zur Verfügung stand, und der zweite aus der korrekten Form und zwei Distraktoren bestand, z. B.

\footnotetext{
${ }^{4}$ Zum eingesetzten Fragebogen siehe den Anhang des vorliegenden Beitrags.

${ }^{5}$ Im Anhang steht die Anleitung für das Ausfüllen des Fragebogens übersetzt ins Deutsche.

${ }^{6}$ In den meisten Fällen war es das Griechische.
} 
ums Leben a.gehen b.kommen c.genießen

Der zweite Teil des Fragebogens (Aufgabe B) sollte - ebenso in Form einer Multiple-ChoiceAufgabe - die korrekte Bedeutungserschließung erfassen, z. B. beim Phrasem ums Leben kommen:

$$
\text { a.geboren werden b.sterben c.überleben }
$$

Ziel des Einsatzes des beschriebenen Fragebogens war, die Phrasemkenntnis der befragten Studierenden in Form und Inhalt zu erheben und die gezeigte Performanz vor dem Hintergrund der verwendeten Lernmaterialien und der geltenden institutionellen Richtlinien kritisch $\mathrm{zu}$ betrachten. Es wurde davon ausgegangen, dass sich die defizitäre Lage des institutionellen Rahmens und der eingesetzten Lehrwerke auf die Entwicklung der phraseologischen Kompetenz von Germanistik-Studierenden negativ auswirkt.

\subsection{Die Datenauswertung}

Die Durchführung der Befragung dauerte etwa 45 Minuten und erfolgte reibungslos. Es folgt die Auswertung der ausgefüllten Fragebögen, wobei die gewonnenen Daten erläutert und graphisch dargestellt werden. Das Alter der befragten Studierenden stellt mit 20,46 Jahren einen zu erwartenden Durchschnitt dar, wobei nur sechs von ihnen über dem Alter von 28 Jahren lagen. $61 \%$ der Studierenden befanden sich zum Zeitpunkt der Erhebung im ersten und $13 \%$ im dritten Studiensemester (s. Tab. 1). Insgesamt befanden sich knapp drei Viertel der befragten Studierenden (74 \%) im Grundstudium, während etwa ein Viertel von ihnen (26\%) Vorlesungen des Grundstudiums nachholte. $64 \%$ der Teilnehmenden befanden sich zum Zeitpunkt der Befragung auf dem B-Niveau und $34 \%$ auf dem C-Niveau. Insbesondere verfügte die Mehrheit der Befragten (39\%) über das B2-Niveau, $25 \%$ über das B1-Niveau, $24 \%$ über das C1-Niveau, $9 \%$ über das C2-Niveau, und $2 \%$ über das A2-Niveau (s. Tab. 2). Dazu sollte angemerkt werden, dass besonders Studierende mit einem deutschen Schulabschluss (dazu s. Abb. 10 und 11), häufig über keine Zertifikate von Sprachkenntnissen verfügen, so dass sie für die Zwecke der vorliegenden Untersuchung dem C2-Niveau zugeordnet wurden. Dies gilt in deutlich geringerem Maße für Studierende, die die Schule in Griechenland absolvierten.
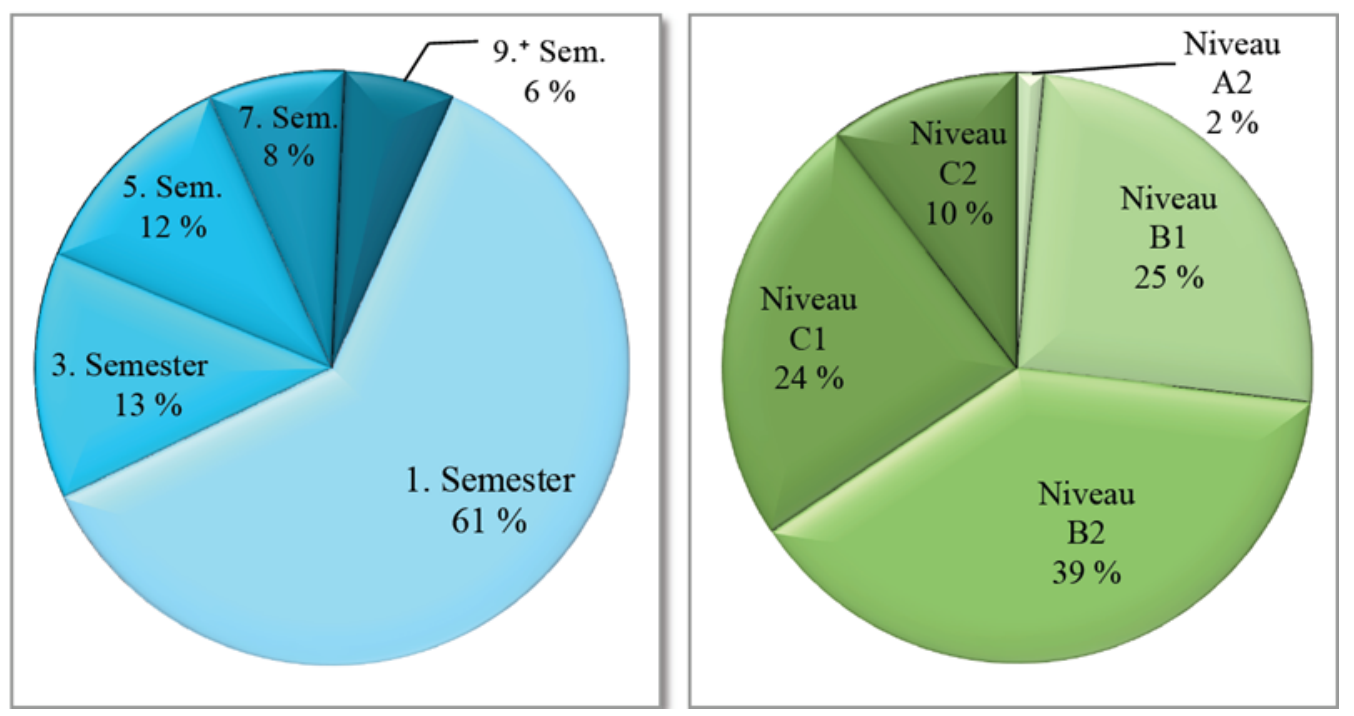

Tab. 1: Studiensemester der befragten Studierenden/Tab. 2: Sprachniveau der befragten Studierenden 
Der eigentliche Teil der Erhebung hatte zunächst die Untersuchung der Kenntnis der Phrasemform (Aufgabe A) zum Ziel. In der ersten Spalte der folgenden Tabelle (s. Tab. 3) werden die zwanzig häufigsten und geläufigsten Phraseme nach Hallsteinsdóttir et al. (2006) dargestellt und in der zweiten Spalte der Prozentsatz der Studierenden angegeben, die das phraseologische Formativ bei den zwanzig vorgegebenen Phrasemen anhand einer Multiple-ChoiceAufgabe richtig vervollständigten. Es fällt auf, dass die Werte im Hinblick auf die Bekanntheit der untersuchten Phraseme weit auseinander liegen. Während 97,01 \% der befragten Studierenden das Phrasem eine Rolle spielen richtig erkannten, erfasste nur etwa jeder fünfte Studierende $(22,39 \%)$ das Phrasem rote Zahlen schreiben korrekt. Zudem wird aus den gewonnenen Daten ersichtlich, dass dreizehn von den insgesamt zwanzig Phrasemen, d. h. fast zwei Drittel davon, von mehr als der Hälfte der Probanden richtig ergänzt wurde.

Ferner zielte die Erhebung auf die Untersuchung der Kenntnis der Phrasembedeutung ab (Aufgabe B). Die Auswertung dieses Aspekts der Phrasemkenntnis korrelierte mit der Auswertung der Angaben in Aufgabe A des Fragebogens. Dabei wurde davon ausgegangen, dass das Erkennen des phraseologischen Formativs und somit der Nennform des Phrasems (Aufgabe A) notwendige Voraussetzung für die Bedeutungserschließung (Aufgabe B) ist. Aus diesem Grund wurde zur Vermeidung von Zufallstreffern methodisch so vorgegangen, dass die korrekten Angaben zur Semantik nur dann in die Datenauswertung einflossen, wenn auch die Phrasemform richtig erkannt worden war, wobei auf eine gesonderte Auswertung der semantisch korrekt erschlossenen Phraseme verzichtet wurde. In der letzten Spalte der folgenden Tabelle (s. Tab. 3) wird der Prozentsatz der Studierenden angegeben, der die zwanzig vorgegebenen Phraseme in Form und Bedeutung richtig erfasste.

\begin{tabular}{|c|c|c|c|}
\hline Phrasem & $\begin{array}{c}\text { Form } \\
\text { korrekt er- } \\
\text { kannt in \% }\end{array}$ & Phrasem & $\begin{array}{l}\text { Form und Be- } \\
\text { deutung korrekt } \\
\text { erkannt in \% }\end{array}$ \\
\hline eine Rolle spielen & 97,01 & eine Rolle spielen & 82,09 \\
\hline ums Leben kommen & 86,57 & $\begin{array}{c}\text { etw. in der Tasche ha- } \\
\text { ben }\end{array}$ & 53,73 \\
\hline $\begin{array}{c}\text { nach vielem Hin und } \\
\text { Her }\end{array}$ & 85,07 & $\begin{array}{c}\text { jmdn/etw. aus den Au- } \\
\text { gen verlieren }\end{array}$ & 50,75 \\
\hline $\begin{array}{c}\text { jmdn/etw. aus den } A u- \\
\text { gen verlieren }\end{array}$ & 73,13 & $\begin{array}{l}\text { nach vielem Hin und } \\
\text { Her }\end{array}$ & 50,75 \\
\hline in der Luft schweben & 71,64 & etw. liegt auf der Hand & 50,75 \\
\hline $\begin{array}{l}\text { etw.jjmdn in Kauf neh- } \\
\text { men }\end{array}$ & 68,66 & grünes Licht geben & 49,25 \\
\hline auf der Straße sitzen & 65,67 & $\begin{array}{l}\text { jmdn unter Druck set- } \\
\text { zen }\end{array}$ & 46,27 \\
\hline etw. liegt auf der Hand & 65,67 & etw. liegt in der Luft & 44,78 \\
\hline $\begin{array}{l}\text { etw. in der Tasche ha- } \\
\text { ben }\end{array}$ & 64,18 & in der Luft schweben & 44,78 \\
\hline etw. liegt in der Luft & 61,19 & $\begin{array}{c}\text { etw. über die Bühne } \\
\text { bringen }\end{array}$ & 43,28 \\
\hline grünes Licht geben & 55,22 & auf der Straße sitzen & 37,31 \\
\hline etw. im Auge behalten & 53,73 & etw. im Auge behalten & 37,31 \\
\hline $\begin{array}{c}\text { etw. über die Bühne } \\
\text { bringen }\end{array}$ & 52,24 & ums Leben kommen & 32,84 \\
\hline
\end{tabular}




\begin{tabular}{|c|c|c|c|}
\hline jmdn unter Druck setzen & 47,76 & $\begin{array}{c}\text { etw./jmdn in Kauf neh- } \\
\text { men }\end{array}$ & 31,34 \\
\hline $\begin{array}{c}\text { etw. auf den Punkt brin- } \\
\text { gen }\end{array}$ & 47,76 & $\begin{array}{c}\text { jmdn/etw. im Griff ha- } \\
\text { ben }\end{array}$ & 31,34 \\
\hline $\begin{array}{c}\text { etw. in den Griff be- } \\
\text { kommen }\end{array}$ & 40,30 & $\begin{array}{c}\text { etw. auf den Punkt } \\
\text { bringen }\end{array}$ & 31,34 \\
\hline $\begin{array}{c}\text { jmdn/etw. im Griff ha- } \\
\text { ben }\end{array}$ & 37,31 & $\begin{array}{c}\text { etw. in den Griff be- } \\
\text { kommen }\end{array}$ & 28,36 \\
\hline in die Luft gehen & 35,82 & $\begin{array}{c}\text { jmdn auf die Straße } \\
\text { setzen }\end{array}$ & 19,40 \\
\hline $\begin{array}{c}\text { jmdn auf die Straße set- } \\
\text { zen }\end{array}$ & 34,33 & in die Luft gehen & 13,43 \\
\hline rote Zahlen schreiben & 22,39 & rote Zahlen schreiben & 10,45 \\
\hline
\end{tabular}

Tab. 3: Erkennen von Form und Bedeutung der Phraseme

Wie zu erwarten war, erreichten die befragten Studierenden insgesamt niedrigere Werte hinsichtlich der Kenntnis von Form und Semantik im Vergleich zur bloßen Kenntnis der Form. Somit ist eine auffällige Diskrepanz zwischen den Erhebungsdaten zur Phrasemkenntnis in Form (Aufgabe A) und zur Phrasemkenntnis in Form und Bedeutung (Aufgabe A und B) zu konstatieren. Nur fünf Phraseme konnten von mehr als der Hälfte der Befragten sowohl formal als auch semantisch zugeordnet werden. So wurde etwa das häufigste und geläufigste Phrasem eine Rolle spielen von 97,01\% der Probanden richtig erkannt, während es nur $82,09 \%$ von ihnen auch in seiner Semantik korrekt erschlossen. Dabei ergibt sich eine Abweichung von $14,02 \%$. Ähnliches gilt für das am wenigsten bekannte Phrasem rote Zahlen schreiben, dessen korrekte Form 22,39 \% der Probanden erkannte, während es 10,45 \% von ihnen der korrekten Bedeutung zuordneten, so dass sich hierbei eine Differenz von 11,94 \% ergibt. Insgesamt variiert die Diskrepanz zwischen korrekter Form einerseits und korrekter Form und Bedeutung andererseits relativ stark. Die geringste Abweichung (1,49\%) ergibt sich für das Phrasem Druck auf jmdn ausüben und die größte $(53,73 \%)$ für das Phrasem ums Leben kommen, das die überwiegende Mehrheit der Probanden vermutlich aufgrund der Inter-

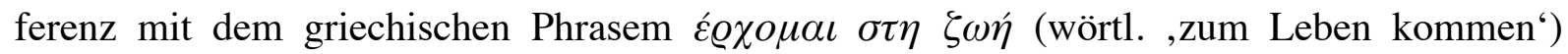
fälschlicherweise der Bedeutung ,geboren werden` zuordnete. Daraus geht hervor, dass die formale Kenntnis von Phrasemen nicht mit der Kenntnis ihrer Bedeutung gleichzusetzen ist. In der folgenden Tabelle (Tab. 4) wird die Diskrepanz zwischen korrekter Form (Aufgabe A) und korrekter Form und Bedeutung (Aufgabe A und B) in aufsteigender Form festgehalten.

\begin{tabular}{|c|c|}
\hline Phrasem & $\begin{array}{c}\text { Diskrepanz zwischen der } \\
\text { Phrasemkenntnis in Form } \\
\text { und der Phrasemkenntnis in } \\
\text { Form und Bedeutung in \% }\end{array}$ \\
\hline jmdn unter Druck setzen & 1,49 \\
\hline grünes Licht geben & 5,97 \\
\hline jmdn/etw. im Griff haben & 5,97 \\
\hline
\end{tabular}


Phraseme des Deutschen

\begin{tabular}{|c|c|}
\hline etw. über die Bühne bringen & 8,96 \\
\hline etw. in der Tasche haben & 10,45 \\
\hline etw. in den Griff bekommen & 11,94 \\
\hline etw. liegt auf der Hand & 11,94 \\
\hline rote Zahlen schreiben & 11,94 \\
\hline eine Rolle spielen & 14,02 \\
\hline jmdn auf die Straße setzen & 14,93 \\
\hline etw. liegt in der Luft & 16,41 \\
\hline etw. im Auge behalten & 16,42 \\
\hline etw. auf den Punkt bringen & 16,42 \\
\hline jmdn/etw. aus den Augen verlieren & 22,38 \\
\hline in die Luft gehen & 22,39 \\
\hline in der Luft schweben & 26,86 \\
\hline auf der Straße sitzen & 28,36 \\
\hline nach vielem Hin und Her & 34,32 \\
\hline etw./jmdn in Kaufnehmen & 37,32 \\
\hline ums Leben kommen & 53,73 \\
\hline
\end{tabular}

Tab. 4: Diskrepanz zwischen der Kenntnisbereiche

In der phraseodidaktischen Literatur wird häufig auf die Relevanz des muttersprachlichen Wissens für die Erarbeitung fremdsprachlicher Phraseme hingewiesen. Hessky (1997: 142) und Jesenšek (2007: 22) heben den fundamentalen Einfluss der Muttersprache auf das Erkennen und Verstehen von Phrasemen hervor, wobei Hallsteinsdóttir (2001: 300) die Relevanz der Muttersprache für die Rezeption von Phrasemen in einer fragebogengeleiteten Studie empirisch belegt. Diese These bestätigt auch die Performanz der befragten Studierenden in der vorliegenden Erhebung. So weisen sechs von den ersten sieben Phrasemen in der oben stehenden Tabelle (s. Tab. 4), d. h. knapp etwa ein Drittel der untersuchten Wendungen, bei denen die befragten Studierenden die höchsten Werte erreichen, formal und semantisch äquivalente Phraseme im Griechischen auf, da sie sich interlingual in Hinblick auf lexikalische Besetzung und Morphosyntax entsprechen. ${ }^{7}$ Im Gegensatz dazu weisen die letzten sieben Phraseme in der oben stehenden Tabelle, d. h. gut ein Drittel der untersuchten Phraseme, eine hohe Fehlerquote auf, nicht zuletzt, weil sie im Griechischen entweder über kein phraseologisches Äquivalent oder über partielle Entsprechungen mit verschiedener lexikalischer Beset-

\footnotetext{
${ }^{7}$ Eine Ausnahme bildet hier das Phrasem etw. liegt auf der Hand, das ein relativ hoher Prozentsatz der Probanden trotz fehlenden phraseologischen Äquivalents mit ähnlicher Motivationsbasis im Griechischen formal und semantisch korrekt erkennt. Hier wirkt sich vermutlich die semantische Transparenz positiv auf das korrekte Erkennen des Phrasems aus.
} 
zung verfügen. Dies zeigt den signifikanten Einfluss der muttersprachlichen Phraseologie auf das Erkennen äquivalenter fremdsprachlicher Phraseme aufgrund des positiven Transfers.

Da die Probanden keineswegs eine homogene Gruppe darstellen, wird ihre Leistung im Folgenden unter verschiedenen Aspekten analysiert. Zunächst wird der Zusammenhang zwischen korrekten Angaben und Sprachniveau beleuchtet. Die befragten Studierenden auf dem B1Niveau erkannten etwas weniger als die Hälfte der Phraseme in ihrer Form und erschlossen etwas mehr als ein Viertel der Phraseme auch semantisch korrekt. Die Probanden auf dem B2-Niveau, die auch die Mehrheit der Befragten darstellen, erkannten und entschlüsselten um 5-7 \% korrekter die vorgegebenen Phraseme im Vergleich zu den Studierenden auf dem B1Niveau. Um 9-14 \% bessere Werte als die Probanden auf dem B2-Niveau erreichten die Probanden auf dem C1-Niveau. Wie zu erwarten war, zeigten die Studierenden auf dem C2Niveau die beste Leistung: Fast $85 \%$ von ihnen konnten die Phraseme hinsichtlich ihrer Form korrekt erkennen, wobei $75 \%$ vom ihnen die Phraseme auch hinsichtlich ihrer Bedeutung richtig entschlüsselte. Im folgenden Diagramm (s. Tab. 5) sind die ermittelten Werte graphisch abgebildet: ${ }^{8}$

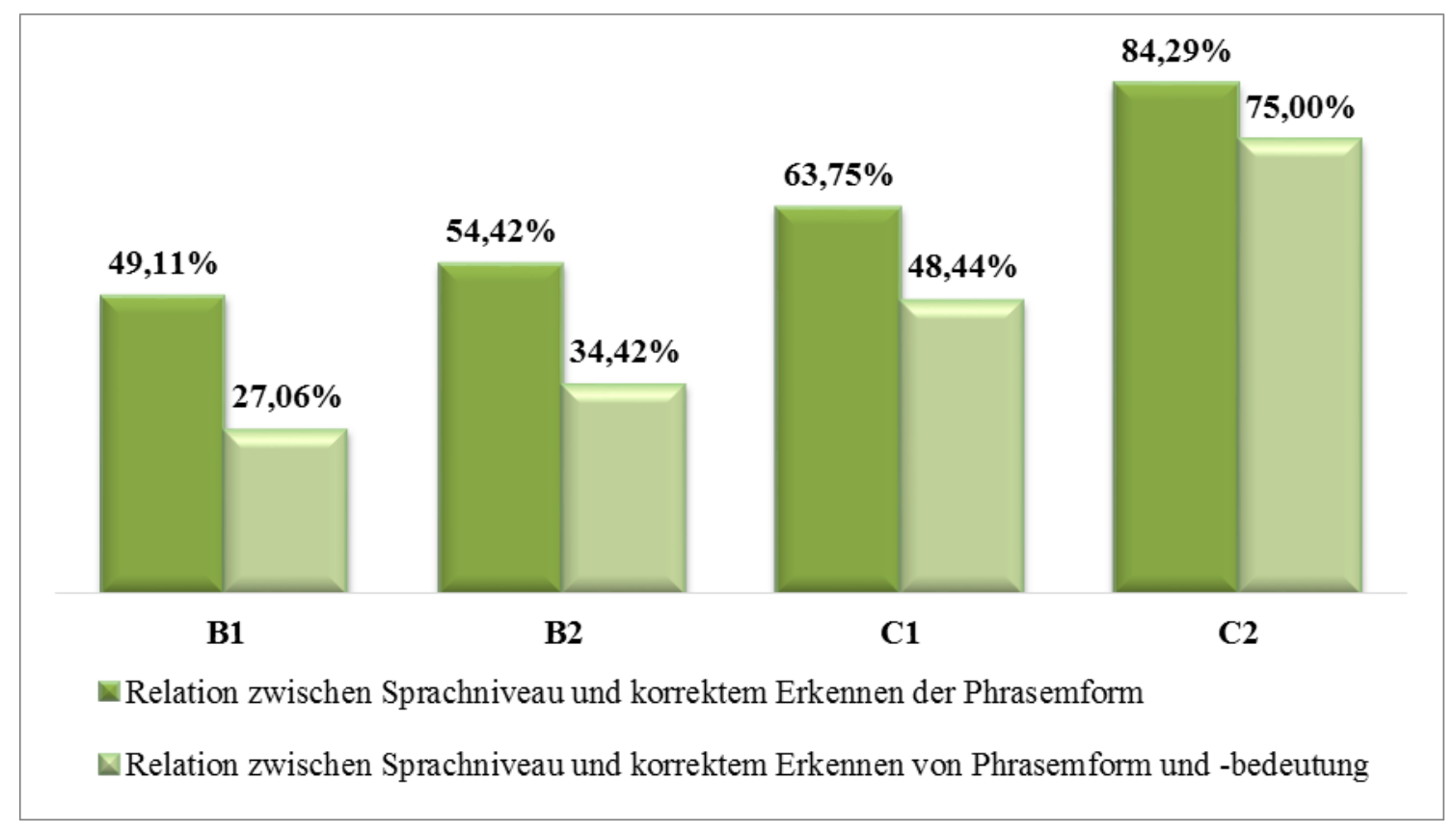

Tab. 5: Relation zwischen Sprachniveau und korrektem Erkennen der Phraseme

Bedeutsam für die Interpretation der Performanz der befragten Studierenden ist nicht zuletzt die Umgebung ihrer Sozialisation. Die Mehrheit der Probanden ist in Griechenland mit Griechisch als Muttersprache aufgewachsen, gefolgt von in Griechenland aufgewachsenen Studierenden mit Deutsch als Mutter- bzw. Zweitsprache und von in Deutschland sozialisierten Probanden mit Deutsch als Mutter- bzw. Zweitsprache. Wie die folgende Tabelle (s. Tab. 6) veranschaulicht, stammt die höchste Leistung von Studierenden, die in Deutschland sozialisiert wurden. Es folgen Studierende, die in Griechenland mit Deutsch in der Familie aufgewachsen sind und schließlich Studierende, die in Griechenland mit Griechisch als Muttersprache sozialisiert wurden.

\footnotetext{
8 Aufgrund des nicht signifikanten Prozentsatzes der Studierenden (2\%), die das A2-Niveau angeben, wird hierbei davon abgesehen.
} 


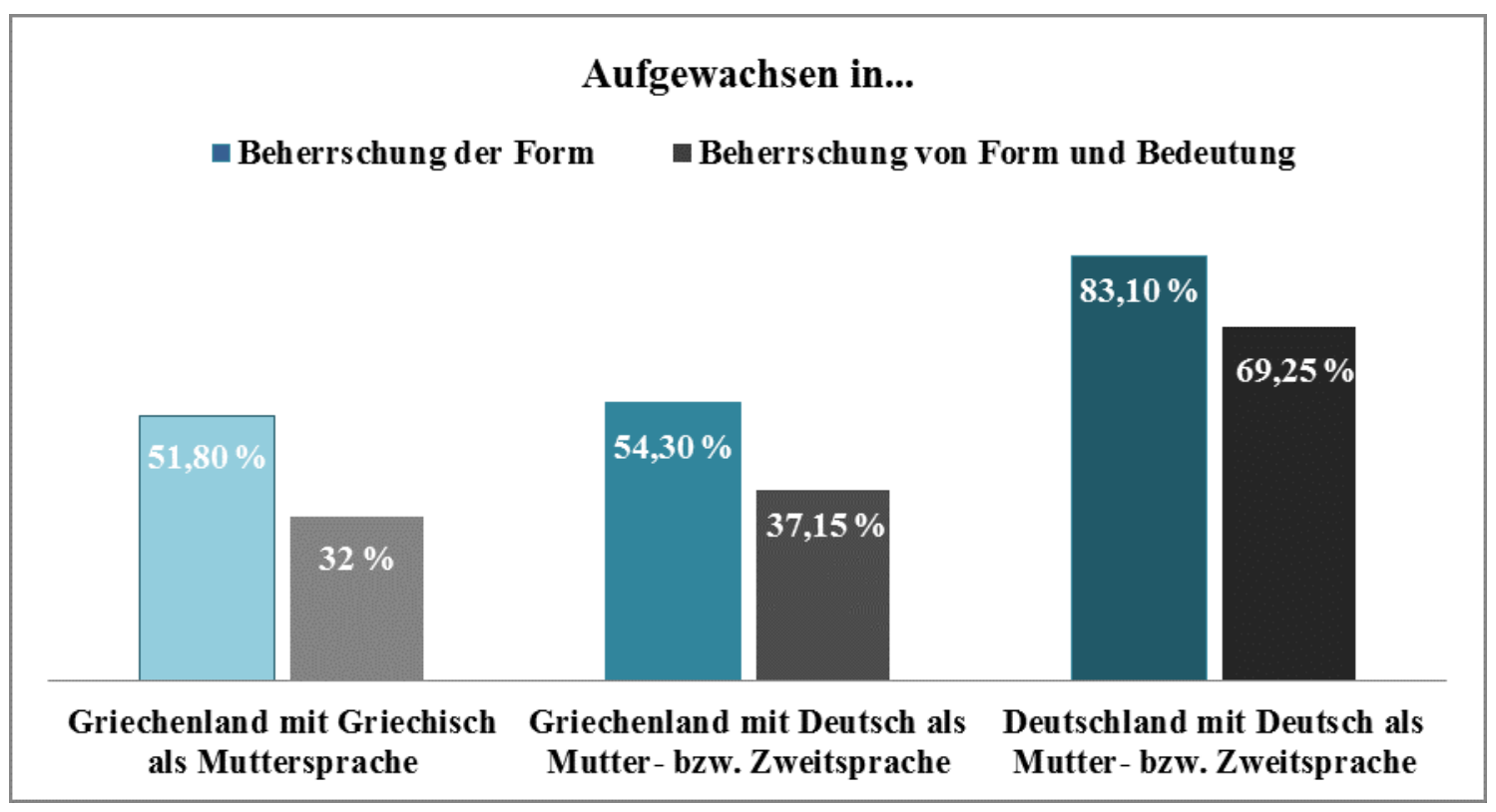

Tab. 6: Umgebung der Sozialisation der befragten Studierenden

Die Studierenden gaben ferner an, Deutsch mit einer Vielfalt von Lernmaterialien zu lernen bzw. gelernt zu haben, die im Folgenden (s. Tab. 7) nach der Häufigkeit der Nennung abgebildet sind. ${ }^{9}$ Es ist davon auszugehen, dass die wenig zufriedenstellenden Ergebnisse der vorliegenden Erhebung nicht zuletzt vor dem Hintergrund der genannten Lernmaterialien zu interpretieren sind, die - wie die Mehrheit der Lernmaterialien für Deutsch als Fremdsprache ein kaum fundiertes Vorgehen bei der Auswahl, Präsentation und didaktischen Aufbereitung von Phrasemen aufweisen. ${ }^{10}$

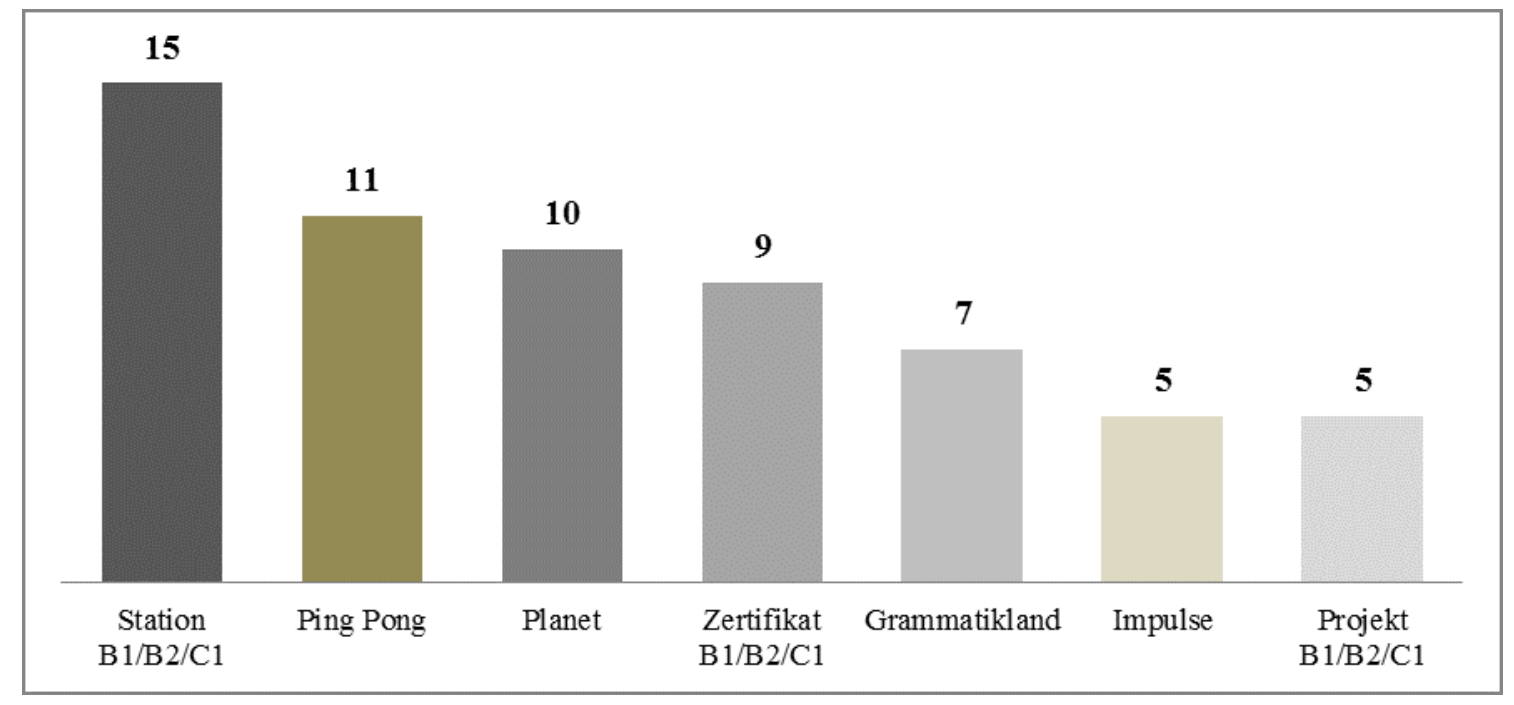

Tab. 7: Lernmaterialien, mit denen die befragten Studierenden Deutsch lernten bzw. lernen

\footnotetext{
${ }^{9}$ Hierbei wird aufgrund der nicht signifikanten Menge von Lehrwerken abgesehen, die seltener als fünfmal genannt wurden.

${ }^{10}$ Dies bestätigen die Befunde von Winzer-Kiontke (2016), die vier gängige Lehrwerke für Deutsch als Fremdsprache systematisch auf das Vorkommen von Routineformeln hin analysiert.
} 


\section{$6 \quad$ Fazit}

Die Notwendigkeit der gezielten und systematischen Spracharbeit im Bereich der Phraseologie stellt besondere Anforderungen an die Gestaltung der Wortschatzarbeit im Unterricht Deutsch als Fremdsprache, die einer Steuerung auf der Ebene von institutionellen Richtlinien, Lernmaterialien und didaktischen Verfahren bedarf. Dies geht auch aus der fragebogengeleiteten Erhebung der Phrasemkenntnis bei Germanistik-Studierenden an der Nationalen Kapodistrias-Universität Athen hervor.

Aus der durchgeführten Erhebung geht zunächst hervor, dass das Wissen um die korrekte Form eines Phrasems keineswegs auch die Kenntnis seiner Bedeutung impliziert. Zwischen diesen zwei Größen herrscht eine signifikante Diskrepanz, die aus didaktischer Sicht die gezielte Festigung von Phrasemform und -bedeutung nahelegt. Außerdem lässt sich ein enger Zusammenhang zwischen der Phrasemkenntnis auf formaler und semantischer Ebene und dem zertifizierten Sprachniveau feststellen, da fortgeschrittene Probanden auch im Bereich der Phraseologie bessere Leistungen erbringen. Ferner zeigt sich, dass sich die interlinguale Konvergenz positiv auf die Leistung der Probanden auswirkt. Dies legt die gezielte Einbeziehung der interlingualen Perspektive und die Adaption des phraseologischen Grundwortschatzes auf die Muttersprache der Lernenden in regionalen Lernmaterialien für Deutsch als Fremdsprache nahe. Nicht zuletzt nimmt die Umgebung der Sozialisation Einfluss auf die Performanz der Studierenden: Während die in deutscher Umgebung aufgewachsenen Studierenden die beste Leistung erbringen, zeigen die in griechischer familiärer Umgebung sozialisierten Probanden signifikante Schwankungen in ihrer Leistung, die mit ihrem Sprachniveau zusammenhängen.

Aus der Datenanalyse wird insgesamt ersichtlich, dass die Phrasemkenntnis der befragten Studierenden wenig ausgeprägt und durchaus ausbaufähig ist. Bedenkt man, dass es sich bei den ausgewählten Phrasemen um kommunikativ wichtige Phraseme des Deutschen handelt, die im Sprachgebrauch besonders frequent vorkommen und eine hohe Geläufigkeit aufweisen, sind die erreichten Werte insgesamt kaum als befriedigend einzustufen. Einerseits lassen sie sich auf die vagen institutionellen Richtlinien (Gemeinsamer Europäischer Referenzrahmen für Sprachen, Profile Deutsch, Lehrpläne) zurückführen, die den Lernzuwachs bei der sukzessiven Entwicklung der phraseologischen Kompetenz kaum systematisch erfassen und die Lernprogression im Sinne einer nachvollziehbaren zeitlichen Anordnung erwerbsrelevanter phraseologischer Lexik auf den einzelnen Sprachniveaus nicht differenziert genug beschreiben. Andererseits ist die verbesserungsbedürftige Leistung der befragten Studierenden nicht zuletzt auf die kaum systematische Behandlung phraseologischer Phänomene in Lernmaterialien zurückzuführen.

Einschränkend zur Gültigkeit der vorliegenden Untersuchung ist anzumerken, dass es sich dabei um keine Längsschnittstudie handelt und dass die instrumentalisierten Multiple-ChoiceAufgaben im Fragebogen Zufallstreffer naturgemäß nicht ausschließen. Bei der durchgeführten Erhebung handelt es sich um eine qualitative Bestandsaufnahme, die Tendenzen im Bereich der phraseologischen Kompetenz von Germanistik-Studierenden aufzeigt und deren Ergebnisse sich zukünftig auf der Grundlage von Langzeitstudien mit größeren Stichproben verifizieren lassen. 
Aus der durchgeführten Untersuchung lassen sich Anschlusshandlungen für die Gestaltung der Wortschatzarbeit auf institutioneller und didaktischer Ebene ableiten, die dringliche phraseodidaktische Desiderate darstellen: Auf institutioneller Ebene ist eine Aktualisierung des Gemeinsamen Europäischen Referenzrahmens für Sprachen durch die transparente Beschreibung der sukzessiven Entwicklung der phraseologischen Kompetenz anhand einer präziseren Beschreibung von Lernzielen und Lerninhalten in ihrer zeitlichen Abfolge zu leisten. Voraussetzung dafür ist laut Konecny et al. (2013: 163) die Klärung der Kriterien, die für eine Niveauzuordnung von Phrasemen zu berücksichtigen sind. Für die Ersteller von Lernmaterialien für Deutsch als Fremdsprache sind damit Entscheidungen verbunden, die die begründete Selektion eines Ausschnitts erwerbsrelevanter phraseologischer Lexik und ihrer Anordnung in einer sinnvollen Lernprogression sowie ihre prozess-, handlungs- und textorientierte didaktische Aufbereitung in Anlehnung an die Ergebnisse der phraseodidaktischen Forschung involvieren. ${ }^{11}$ Wertvolle Dienste in dieser Richtung kann der - vorwiegend idiomatische - phraseologische Grundwortschatz von Hallsteinsdóttir et al. (2006) sowie die in Profile Deutsch erfasste - vorwiegend nicht idiomatische - phraseologische Lexik im Sinne eines offenen, erweiterbaren Grundwortschatzes leisten. Wünschenswert ist ebenfalls das Verfassen von Landescurricula $^{12}$ in Anlehnung an die Forschungsergebnisse der Phraseodidaktik, die den Stellenwert der Phraseologie im mündlichen und schriftlichen Sprachgebrauch herausstellen und einen brauchbaren Rahmen für die effiziente Gestaltung der Wortschatzarbeit in diesem Bereich bieten.

Wichtig in diesem Zusammenhang ist schließlich eine angemessene Lehrerausbildung, die auf die Entwicklung eines sprachwissenschaftlich fundierten Bewusstseins für phraseologische Phänomene abzielt und didaktische Zugriffe für die systematische Wortschatzarbeit bereitstellt. Grundlegender Bestandteil der Lehrerausbildung sollte die kritische Auseinandersetzung mit Beispielen guter Praxis sein, die über die traditionelle Lehrwerkarbeit hinausgehen. Dazu gehören Lernangebote wie das mehrsprachige digitale Lernmaterial von EPHRAS (2006) sowie das Online-Lernangebot von Ettinger (2009) und Bergerová (s. a.).

\section{Literatur}

Albert, Ruth/Marx, Nicole (2014): Empirisches Arbeiten in Linguistik und Sprachlehrforschung: Anleitung zu quantitativen Studien von der Planungsphase bis zum Forschungsbericht. 2. Auflage. Tübingen: Narr.

Bergerová, Hana (s.a.): Multimediales Unterrichtsmaterial zur deutschen Phraseologie. http://frazeologie.ujepurkyne.com [24.11.2017].

Bubenhofer, Noah/Ptashnyk, Stefaniya (2010): „Korpora, Datenbanken und das Web: State of the Art computergestützter Forschung in der Phraseologie und Lexikographie“. In: Ptashnyk, Stefaniya/Hallsteinsdóttir, Erla/Bubenhofer, Noah (eds.): Korpora Web und Da-

\footnotetext{
${ }^{11}$ Siehe hierzu auch die Empfehlungen an Autoren von Lehrwerken in Winzer-Kiontke (2016: 307-311), die sich - speziell mit Blick auf die Routineformeln - auf ihre Auswahl, Anzahl und Verteilung im Lehrwerk, ihre didaktisch-methodische Aufbereitung sowie die Verstehens- bzw. Anwendungsbefähigung beziehen.

12 Vorteile sind in diesem Zusammenhang in einem gemeinsamen Rahmencurriculum für die Fremdsprachen oder sogar auch für die Muttersprache der Lernenden - zu sehen, das von der Einheit des sprachlichen Lernens ausgeht und bereits beim frühen Lernen phraseologische Phänomene in einzel- und zwischensprachlicher Hinsicht ins Bewusstsein der Lerner rückt.
} 
tenbanken. Computergestützte Methoden in der modernen Phraseologie und Lexikographie. Baldmannsweiler, Schneider: 7-19. (= Phraseologie und Parömiologie 25).

Burger, Harald et al. (2007): „Phraseologie: Objektbereich, Terminologie und Forschungsschwerpunkte“. In: Burger, Harald et al. (eds.): Phraseologie. Ein internationales Handbuch zeitgenössischer Forschung. Berlin/New York, de Gruyter: 1-10.

Chrissou, Marios (2012): Phraseologismen in Deutsch als Fremdsprache. Theoretische Grundlagen und Umsetzung eines korpusorientierten Ansatzes. Hamburg: Dr. Kovač.

Chrissou, Marios (i. E.): „Der Stellenwert phraseologischer Lexik im Gemeinsamen Europäischen Referenzrahmen für Sprachen und in ,Profile Deutsch': Hinweise für die Unterrichtspraxis am Beispiel von Deutsch als Fremdsprache“. In: Zybatow, Lew et al. (eds.): Beiträge des 50. Linguistischen Kolloquiums, Innsbruck, 3.-5.9. 2015. Frankfurt a. M. etc., Lang.

Ďurčo, Peter (2007): „Phraseologie und allgemeines Konzept für eine komplex strukturierte Datenbank“. In: Jesenšek, Vida/Fabčič, Melanija (eds.): Phraseologie kontrastiv und didaktisch. Neue Ansätze in der Fremdsprachenvermittlung. Maribor, Slavistično društvoFilozofska fakulteta: 169-180 (= Zora 47).

EPHRAS (2006): Ein mehrsprachiges phraseologisches Lernmaterial. Mit CD-ROM. Ljubljana: Založba Rokus.

Ettinger, Stefan (2007): „Phraseme im Fremdsprachenunterricht“. In: Burger, Harald et al. (eds.): Phraseologie. Ein internationales Handbuch zeitgenössischer Forschung. Band B. Berlin/New York, de Gruyter: 893-909.

Ettinger, Stefan (2009): Phraseologie und Phraseodidaktik. Übungen. www.ettingerphraseologie.de/pages/deutsche-redewendungen/uebungen.php [24.11.2017].

Europarat (2001): Gemeinsamer europäischer Referenzrahmen für Sprachen: Lernen, lehren, beurteilen. Berlin etc.: Langenscheidt.

Glaboniat, Manuela et al. (2005): Profile deutsch. Gemeinsamer europäischer Referenzrahmen. Lernzielbestimmungen, Kannbeschreibungen, kommunikative Mittel. Niveau A1-A2, B1-B2, C1-C2. Berlin: Langenscheidt.

Hallsteinsdóttir, Erla (2001): Das Verstehen idiomatischer Phraseologismen in der Fremdsprache Deutsch. Hamburg: Dr. Kovač.

Hallsteinsdóttir, Erla/Šajánková, Monika/Quasthoff, Uwe (2006): „Phraseologisches Optimum für Deutsch als Fremdsprache. Ein Vorschlag auf der Basis von Frequenz- und Geläufigkeitsuntersuchungen“. In: Linguistik Online 27/2: 117-136.

Hessky, Regina (1997): „Feste Wendungen - Ein heißes Eisen? Einige phraseodidaktische Überlegungen für den DaF-Unterricht“. In: Deutsch als Fremdsprache 34/3: 139-143.

Hessky, Regina (2007): „Perspektivenwechsel in der Arbeit mit Phraseologie im DaFUnterricht“. In: Jesenšek, Vida/Fabčič, Melanija (eds.): Phraseologie kontrastiv und didaktisch. Neue Ansätze in der Fremdsprachenvermittlung. Maribor, Slavistično društvo, Filozofska fakulteta: 9-16.

Jesenšek, Vida (2007): „Lehr- und Lerngegenstand Phraseologie“. In: Jesenšek, Vida/Fabčič, Melanija (eds.): Phraseologie kontrastiv und didaktisch. Neue Ansätze in der Fremdsprachenvermittlung. Maribor, Slavistično društvo, Filozofska fakulteta: 17-26.

Konecny, Christine/Hallsteinsdóttir, Erla/Kacjan, Brigita (2013): „Zum Status quo der Phraseodidaktik: Aktuelle Forschungsfragen, Desiderata und Zukunftsperspektiven“. In: 
Konecny, Christine/Hallsteinsdóttir, Erla/Kacjan, Brigita (eds.): Phraseologie im Sprachunterricht und in der Sprachendidaktik/Phraseology in language teaching and in language didactics. Bielsko-Biała etc., Mednarodna založba Oddelka za slovanske jezike in knjiŽevnosti: 153-172. (= Zora 94).

Sinclair, John (2004): "New Evidence, New Priorities, New Attitudes". In: Sinclair, John (ed.): How to use Corpora in Language Teaching. Amsterdam etc., Benjamins: 271-300.

Sinclair, John (2008): "Preface”. In: Granger, Sylviane/Meunier, Fanny (eds.): Phraseology. An interdisziplinary perspective. Amsterdam, Benjamins: XV-XIIX.

Steyer, Kathrin (2013): Usuelle Wortverbindungen. Zentrale Muster des Sprachgebrauchs aus korpusanalytischer Sicht. Tübingen: Narr. (= Studien zur Deutschen Sprache 65).

Winzer-Kiontke, Britta (2016): Routineformeln als Lehr- und Lerngegenstand. Eine Untersuchung zu Vorkommen und didaktischer Aufbereitung von Routineformeln in Lehrwerken für Deutsch als Fremd- und Zweitsprache. München: Iudicium.

\section{Anhang}

\section{Fragebogen zum Bekanntheitsgrad fester Wortverbindungen (Phraseme)}

Der vorliegende Fragebogen stellt ein Untersuchungsinstrument im Rahmen des Seminars „Lexikologie und Wortschatzdidaktik“ des Aufbaustudiengangs "Schnittstellen von Linguistik und Sprachdidaktik“ des Fachbereichs für deutsche Sprache und Literatur der Nationalen Kapodistrias-Universität Athen. Der Fragebogen ist anonym auszufüllen. Die Erhebungsdaten werden im Sinne des Datenschutzes ausschließlich für die Belange der vorliegenden Untersuchung genutzt. Wir bitten euch darum, aufrichtig zu antworten.

\section{Kreuze bitte an.}

1. Wie alt bist du? Jahre

2. In welchem Semester bist du?
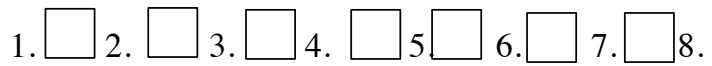

3. Hast du ein Sprachdiplom in Deutsch?

$\mathrm{Ja}$

Nein Wenn ja, welches?

\begin{tabular}{|l|l|l|l|l|l|}
\hline $\mathrm{A} 1$ & $\mathrm{~A} 2$ & $\mathrm{~B} 1$ & $\mathrm{~B} 2$ & $\mathrm{C} 1$ & $\mathrm{C} 2$ \\
\hline
\end{tabular}

4. Bist du in Deutschland aufgewachsen?

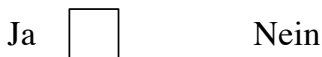

5. Bist du in Griechenland in deiner Familie $\mathrm{Ja}$ Nein mit Deutsch aufgewachsen?

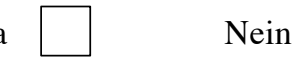

6. Mit welchen Lehrwerken bzw. Lernmaterialien hast du Deutsch gelernt?

\section{Aufgabe A}

\section{Welche der folgenden festen Wortverbindungen (Phraseme) kennst du?}

Wähle bitte die passende Fortsetzung für die folgenden Phraseologismen aus:
1. ums Leben ...
$\square$ gehen
$\square$ kommen
$\square$ genießen
2. auf der Straße ...

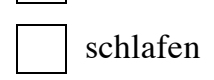
$\square$ essen 
3. jmdn unter Druck ...

\begin{tabular}{|c|c|c|}
\hline legen & stellen & setzen \\
\hline stellen & legen & setzen \\
\hline geben & nehmen & bekommen \\
\hline erleben & geben & spielen \\
\hline ist & liegt & fliegt \\
\hline haben & schweben & verlieren \\
\hline verlieren & verpassen & verachten \\
\hline Hin & Her & Über \\
\hline gehen & reisen & bringen \\
\hline führen & sehen & behalten \\
\hline spielen & bringen & darstellen \\
\hline bekommen & halten & geben \\
\hline anzünden & geben & einschalten \\
\hline halten & haben & führen \\
\hline legen & stellen & bringen \\
\hline liegt & sitzt & nimmt \\
\hline schreiben & notieren & rechnen \\
\hline suchen & haben & holen \\
\hline
\end{tabular}

4. jmdn auf die Straße ...

5. etwas / jmdn in Kauf ...

6. eine Rolle ...

7. etwas ... in der Luft

8. in der Luft ...

9. jmdn / etw. aus den Augen ...

10. nach vielem Hin und ...

11. in die Luft ...

12. etwas im Auge ...

13. etwas über die Bühne ...

14. etwas in den Griff ...

15. grünes Licht ...

16. jmdn / etwas im Griff ...

17. etwas auf den Punkt ...

18. etwas ... auf der Hand

19. rote Zahlen ...

20. etwas in der Tasche ...

\section{Aufgabe B}

Welche der folgenden Bedeutungen entsprechen den obigen festen Wortverbindungen (Phrasemen)?

Markiere bitte das entsprechende Kästchen.

\begin{tabular}{|c|c|c|c|}
\hline 1. & geboren werden & sterben & überleben \\
\hline 2. & auf ein Taxi warten & auf Shoppingtour sein & obdachlos sein \\
\hline 3. & jmdn bedrängen & jmdn umarmen & jmdn festhalten \\
\hline 4. & jmdn schlecht behandeln & jmdn aus dem Haus begleiten & jmdn entlassen \\
\hline 5. & $\begin{array}{l}\text { etw./jmdn zum Einkaufen } \\
\text { mitnehmen }\end{array}$ & etw./jmdn hinnehmen müssen & etw./jmdn austauschen \\
\hline 6. & etw. läuft im Kino & etw. ist wichtig & etw. ist interessant \\
\hline 7. & etw. ist luftig & etw. ist zu erwarten & etw. fliegt durch die Luft \\
\hline 8. & noch nicht entschieden sein & $\begin{array}{l}\text { noch nicht auf den Boden gefal- } \\
\text { len sein }\end{array}$ & schlecht riechen \\
\hline 9. & keinen Kontakt mehr haben & jmdn nicht anschauen & $\begin{array}{l}\text { mit jmdm keinen Augenkontakt ha- } \\
\text { ben }\end{array}$ \\
\hline 10. & $\begin{array}{c}\text { nach vielen Reisen ins Aus- } \\
\text { land }\end{array}$ & $\begin{array}{c}\text { nach einer längeren Phase der } \\
\text { Ungewissheit }\end{array}$ & nach einer großen Pause \\
\hline
\end{tabular}


Marios Chrissou und Evangelos Makos: Zum Bekanntheitsgrad erwerbsrelevanter

Phraseme des Deutschen

\begin{tabular}{|l|c|c|c|}
\hline 11. & abheben & wütend werden & glücklich sein \\
\hline 12. & etw. beobachten & sich an etw. erinnern & sich für etw. entscheiden \\
\hline 13. & eine Szene drehen & auf der Bühne auftreten & etw. erfolgreich durchführen \\
\hline 14. & etw. attackieren & mit etw. umgehen können & nach etw. greifen \\
\hline 15. & den Verkehr regeln & ökologisch handeln & jmdm die Erlaubnis erteilen \\
\hline 16. & jmdn/etw. begriffen haben & die Kontrolle über jmdn/etw. & nach jmdm/etw. mit den Händen \\
\hline 17. & etw. treffend formulieren & einen Punkt setzen & einen Punkt gewinnen \\
\hline 18. & etw. ist zum Verschenken & etw. ist offensichtlich & etw. ist zum Verkaufen \\
\hline 19. & gegen etw. demonstrieren & eine falsche Antwort geben & einen Verlust machen \\
\hline 20. & etw. ganz sicher haben & viel Geld dabei haben & einen Großeinkauf machen \\
\hline
\end{tabular}

Vielen Dank! 